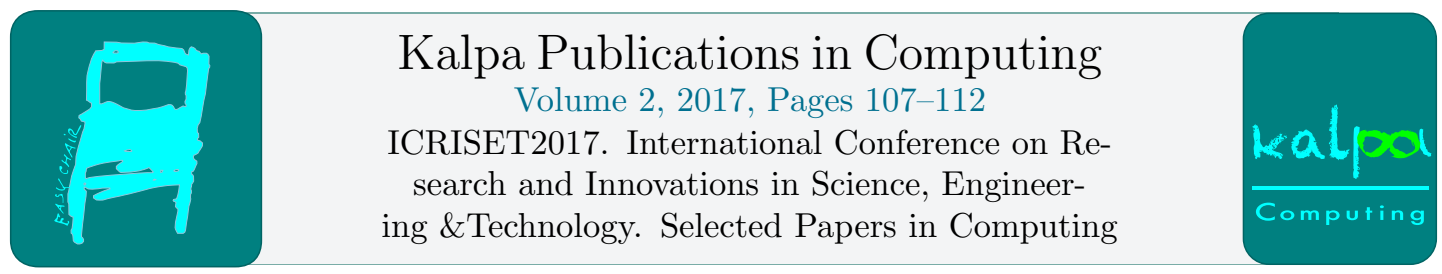

\title{
One-Dimensional Heat Equation Subject to Both Neumann and Dirichlet Initial Boundary Conditions and Used A Spline Collocation Method
}

\author{
Nilesh Patel 1 and Jigisha U. Pandya ${ }^{2}$ \\ ${ }^{1}$ Department of Mathematics, \\ Shankersinh Vaghela Bapu Institute of Technology, Gandhinagar, Gujarat \\ ${ }^{2}$ Department of Mathematics, \\ Sarvajanik college of Engineering \& Technology, Surat, Gujarat \\ napatel1984@gmail.com, jigisha.pandyaescet.ac.in
}

\begin{abstract}
In this paper, one-dimensional heat equation subject to both Neumann and Dirichlet initial boundary conditions is presented and a spline Collocation Method is utilized for solving the problem. also Spline provides continuous solution in contrast to finite difference method, which only provides discrete approximations. It is found that this method is a powerful mathematical tool and can be applied to large class of linear and nonlinear problem in different fields of science and technology. Numerical results obtained by the present method are in a good agreement with the analytical solutions available in the literature.
\end{abstract}

\section{Keywords}

Spline collocation method, Partial differential equations, Heat conductions, Dirichlet and Neumann boundary conditions. 


\section{Introduction}

Recently, new Numerical methods have gained the interest of researchers for finding numerical solutions to partial differential equations. This interest was driven by the need from applications both in industry and in sciences. Theory and numerical methods for solving initial boundary value problems were investigated by many researchers. In the last decade, there has been a growing interest in the new Numerical techniques for linear and nonlinear initial boundary value problems. In the field of partial differential equations, a boundary value problem is a differential equation together with a set of additional restraints, called the boundary conditions. A solution to a boundary value problem is a solution to the differential equation, which also satisfies the boundary conditions. Boundary value problems arise in engineering, applied mathematics and several branches. However, it is usually difficult to obtain closed-form solutions for boundary value problems, especially for nonlinear boundary value problems. In most cases, only approximate solutions (either numerical solution or analytical solutions) can be expected. Some numerical methods such as finite difference method, finite element method, spline approximation method, Jacobi's Method and SOR method have been developed for obtaining numerical solutions to boundary value problems. In this paper, we will apply a spline collocation method approach, to obtain a solution to the wide class of nonlinear systems of boundary value problems. The spline method approach is widely utilized for the numerical solution of nonlinear problems arising in real world applications. The governing equations here are highly partial differential equations, which are solved by using the spline collocation method. In this way, the paper has been organized as follows. In section 2, we use the spline collocation method. Section 3, approximate solution for the governing equations and contains the results and discussion. The conclusions are summarized in section 4 .

\section{Spline collocation method}

Consider parabolic PDE having one space variable

$$
u_{t}=c^{2} u_{x x} 0<x<1, t>0 .
$$

With Dirichlet boundary conditions $\quad \mathrm{u}(0, \mathrm{t})=\mathrm{u}(\mathrm{L}, \mathrm{t})=0$

Along with initial conditions $\mathrm{u}(\mathrm{x}, 0)=\mathrm{f}(\mathrm{x}) ; 0<\mathrm{x}<1$. We divide the region into say n-equal subinterval each of width $\mathrm{h}$. let us denote the points of subdivisions by $\mathrm{x}_{0}, \mathrm{x}_{1}, \ldots . \mathrm{x}_{\mathrm{n} . .}$ let us have the solution on hand at time $\mathrm{j} \Delta \mathrm{t}$ at the mesh points $\mathrm{x}_{0}, \mathrm{x}_{1}, \ldots . \mathrm{x}_{\mathrm{n} . .}$ let denote the value of $\mathrm{u}$ at $\mathrm{i}^{\text {th }}$ mesh point at time $\mathrm{j} \Delta \mathrm{t}$. Now discritizing left side of equation (i) by forward difference formula and replacing right side by the second derivatives $\mathrm{S}^{\text {' ' }}(\mathrm{xi})$ at $\mathrm{j}^{\text {th }}$ level like explicit scheme in finite difference we get

$$
\left(u_{i, j+1}-u_{i, j}\right) / \Delta t=c^{2} S_{i, j}^{\prime \prime}
$$

Where denotes $S_{i, j}^{\prime \prime}\left(x_{i}\right)$ at $j^{\text {th }}$ level substitute values of $S_{i, j}^{\prime \prime}$ in the below equation

$$
S^{\prime \prime-1, j}+S^{\prime \prime, j}+S^{\prime \prime}{ }_{i+1, j}=\left(6 / h^{2}\right)\left(u_{i-1, j}-2 u_{i, j}+u_{i+1, j}\right) \text {. }
$$

Then we get 


$$
\begin{aligned}
& \frac{\left(u_{i-1, j+1}-u_{i-1, j}\right)}{c^{2} \Delta t}+4 \frac{\left(u_{i, j+1}-u_{i, j}\right)}{c^{2} \Delta t}+\frac{\left(u_{i+1, j+1}-u_{i+1, j}\right)}{c^{2} \Delta t} \\
& =\frac{6}{h^{2}}\left[u_{i-1, j}-2 u_{i, j}+u_{i+1, j}\right] \\
& u_{i-1, j+1}+4 u_{i, j+1}+u_{i+1, j+1}=\left(1+6 \frac{c^{2} \Delta t}{h^{2}}\right) u_{i-1, j}+\left(4-12 \frac{c^{2} \Delta t}{h^{2}}\right) u_{i, j}+\left(1+6 \frac{c^{2} \Delta t}{h^{2}}\right) u_{i+1, j} \\
& u_{i-1, j+1}+4 u_{i, j+1}+u_{i+1, j+1}=(1+6 r) u_{i-1, j}+(4-12 r) u_{i, j}+(1+6 r) u_{i+1, j} \ldots \ldots \ldots \ldots \ldots \ldots . . .(v)
\end{aligned}
$$

This set of simultaneous equations can be solved. Equation (v), which is known as cubic spline explicit formula to solve equation (i). The finite difference replacement of (i) corresponding to implicit scheme is

$$
\frac{u_{i, j+1}-u_{i, j}}{\Delta t}=\frac{c^{2}}{2}\left(S_{i, j}^{\prime \prime}+S_{i, j+1}^{\prime \prime}\right) .
$$

Where $S^{\prime \prime}{ }_{i, j}, S^{\prime \prime}{ }_{i, j+1}$ denote second derivative at $x=x_{i}$ the time level $j$ and $j+1$.

$$
\begin{aligned}
& S_{i-1, j}^{\prime \prime}+S_{i, j}^{\prime \prime}+S_{i+1, j}^{\prime \prime}=\frac{6}{h^{2}}\left(u_{i-1, j}-2 u_{i, j}+u_{i+1, j}\right) \\
& S_{i-1, j+1}^{\prime \prime}+S_{i, j+1}^{\prime \prime}+S_{i+1, j+1}^{\prime \prime}=\frac{6}{h^{2}}\left(u_{i-1, j+1}-2 u_{i, j+1}+u_{i+1, j+1}\right)
\end{aligned}
$$

Putting the value $S{ }_{i, j+1}, S{ }_{i+1, j+1}$ and $S{ }^{\prime, j}$ the in (vii) \& (viii)

$$
\begin{aligned}
& \left(2 / c^{2} \Delta t\right)\left(u_{i-1, j+1}-u_{i-1, j}\right)-S_{i-1, j}^{\prime \prime}+4\left[\left(2 / c^{2} \Delta t\right)+\left(u_{i, j}+1-u_{i, j}\right)-S_{i, j}^{\prime \prime}\right] \\
& +\left(2 / c^{2} \Delta t\right)\left(u_{i+1, j+1}-u_{i+1, j}\right)+S_{i+1, j}^{\prime \prime}=\left(6 / h^{2}\right)\left(u_{i-1, j+1}-2 u_{i, j+1}+u_{i+1, j+1}\right) . \\
& (1-3 r) u_{i-1, j+1}+(4+6 r) u_{i, j+1}+(1-3 r) u_{i+1, j+1} \\
& =(1+3 r) u_{i-1, j}+(4-6 r) u_{i, j}+(1+3 r) u_{i+1, j}
\end{aligned}
$$

Above equation ( $\mathrm{x}$ ) is known as cubic spline implicit formula to solve equation (i).Now $\mathrm{u}_{0, \mathrm{j}+1}$ and $\mathrm{u}_{\mathrm{n}, \mathrm{j}+1}$ are known due to the prescribed boundary conditions. The set of simultaneous equations obtained in explicit as well as implicit scheme contains only (n-1) unknowns. These (n-1) equations in (n-1) unknown can be solved by any standard method. The converges and stability of these methods depend upon the value of $r$. For explicit scheme, we have to choose the value of $r$ for $0 \leq r \leq 1$ converges and stability along with small values are more accurate. 


\section{Spline Solutions with Explicit Scheme}

We consider the problem

$$
\mathrm{u}_{\mathrm{t}}=\mathrm{u}_{\mathrm{xx}}, 0<\mathrm{x}<1, \mathrm{t}>0
$$

With initial condition: $\mathrm{u}(\mathrm{x}, 0)=\sin (\pi \mathrm{x})$ and the boundary condition $\mathrm{u}(0, \mathrm{t})=\mathrm{u}(1, \mathrm{t})=0$. Let length is subdivided in into 10 subintervals, we get $\mathrm{h}=\Delta \mathrm{x}=0.1$ and $\Delta \mathrm{t}=0.04$ then we get $\mathrm{r}=0.4$ Substitute the value of $1+6 \mathrm{r}$ and $4-12 \mathrm{r}$ in equation (v) and using initial conditions, we get for $\mathrm{j}=0$

$$
\text { Since } u_{0,1}=0 \begin{aligned}
& i=1, u_{0,1}+4 u_{1,1}+u_{2,1}=1.7512 \\
& 4 u_{1,1}+u_{2,1}=1.7512 \\
& i=2, u_{1,1}+4 u_{2,1}+u_{3,1}=3.3310 \\
& i=3, u_{2,1}+4 u_{3,1}+u_{4,1}=4.5848 \\
& i=4, u_{3,1}+4 u_{4,1}+u_{5,1}=5.3898 \\
& i=5, u_{4,1}+4 u_{5,1}+u_{6,1}=5.6671 \\
& i=6, u_{5,1}+4 u_{6,1}+u_{7,1}=5.3898 \\
& i=7, u_{6,1}+4 u_{7,1}+u_{8,1}=4.5848 \\
& i=8, u_{7,1}+4 u_{8,1}+u_{9,1}=3.3310 \\
& i=9, u_{8,1}+4 u_{9,1}+u_{10,1}=1.7512 \\
& u_{10,1}=0, \quad u_{8,1}+4 u_{9,1}=1.7512
\end{aligned}
$$

Here we get nine algebraic equations in nine unknowns. This system of equation is solved easily by any well-known method. $1-3 r=-0.2,1+3 r=2.2,4+6 r=6.6,4-6 r=2.6$ Substitute the value of $1+3 r$, $1-3 r, 4+6 r$ and 4-6r in equation(x) and using initial conditions, we get for $j=0$

$$
\begin{gathered}
i=1,(-0.2) u_{0,1}+(6.4) u_{1,1}+(-0.2) u_{2,1}=1.7875 \\
\text { Since } u_{0,1}=0 \quad 6.4 u_{1,1}-0.2 u_{2,1}=1.7875 \\
i=2,(-0.2) u_{1,1}+(6.4) u_{2,1}+(-0.2) u_{3,1}=3.4001 \\
i=3,(-0.2) u_{2,1}+(6.4) u_{3,1}+(-0.2) u_{4,1}=4.6798 \\
i=4,(-0.2) u_{3,1}+(6.4) u_{4,1}+(-0.2) u_{5,1}=5.5015 \\
i=5,(-0.2) u_{4,1}+(6.4) u_{5,1}+(-0.2) u_{6,1}=5.7846 \\
i=6,(-0.2) u_{5,1}+(6.4) u_{6,1}+(-0.2) u_{7,1}=5.5015 \\
i=7,(-0.2) u_{6,1}+(6.4) u_{7,1}+(-0.2) u_{8,1}=4.6798 \\
i=8,(-0.2) u_{7,1}+(6.4) u_{8,1}+(-0.2) u_{9,1}=3.4001 \\
i=9,(-0.2) u_{8,1}+(6.4) u_{9,1}+(-0.2) u_{10,1}=1.7815 \\
u_{10,1}=0, \text { then } \\
\quad(-0.2) u_{8,1}+(6.4) u_{9,1}=1.7815
\end{gathered}
$$

Here we get nine algebraic equations in nine unknowns. This system of equation is solved easily by any well-known method. Proceeding in this way, the results obtained by Explicit and Implicit methods are shown in below table. 


\section{Tables}

\begin{tabular}{|c|c|c|c|}
\hline \multicolumn{5}{|c|}{ Comparison of spline solution \& Exact solution } \\
\hline \multicolumn{4}{|c|}{$\mathbf{h}=\Delta \mathbf{x}=\mathbf{0 . 1}, \boldsymbol{\Delta t}=\mathbf{0 . 0 4}$} \\
\hline $\mathbf{X}$ & Explicit Solution & Implicit Solution & Exact solution \\
\hline 0 & 0 & 0 & 0 \\
\hline 0.1 & 0.29707 & 0.29708 & 0.29710 \\
\hline 0.2 & 0.56498 & 0.56499 & 0.56500 \\
\hline 0.3 & 0.77776 & 0.77775 & 0.77777 \\
\hline 0.4 & 0.91419 & 0.91421 & 0.91420 \\
\hline 0.5 & 0.96128 & 0.96131 & 0.96130 \\
\hline 0.6 & 0.91419 & 0.91421 & 0.91420 \\
\hline 0.7 & 0.77776 & 0.77775 & 0.77777 \\
\hline 0.8 & 0.56499 & 0.56500 & 0.56500 \\
\hline 0.9 & 0.29708 & 0.29709 & 0.29710 \\
\hline 1.0 & 0 & 0 & 0 \\
\hline
\end{tabular}

\section{Conclusion}

The aim of this paper has been to find an approximate solution to the one-dimensional heat equation with Dirichlet and Neumann initial boundary conditions using spline explicit and implicit method. The problems solved using the Explicit \& Implicit gave satisfactory results in comparison to those recently obtained by other searchers using exact solution. The case studies gave sufficiently good agreements with the exact solutions. Moreover, the method is easier to implement than the traditional techniques.

\section{References}

[1] CHENIGUEL A(2014):"Numerical Method for the Heat Equation with Dirichlet and Neumann Conditions."Proceedings of the International multi conference of Engineers and Computer Scientists Vol.I, IMECS, March 12 - 14, Hong Kong.

[2] CHENIGUEL A(2011):"Numerical Method for Solving Heat Equation with Derivative Boundary Conditions."Proceedings of the World Congress on Engineering and Computer Science Vol. II, IMECS, October 19-21, USA.

[3] AHLBERG J H, NILSON E N and WALSH J H, "The Theory of Spline and their applications," Academic Press, New York. 
[4] PANDYA J.U.AND DOCTOR H.D.(2010),: “The Numerical Solution of Föppl-Hencky Equation using Spline method", accepted 27 July.

[5] PATHAK A.K. AND DOCTOR H.D,(2009): "Spline Collocation approach to Partial Differential Equation.”Presented in National conf. Gujarat Science Congress, Surat.

[6] BLUE J L, " Spline Function Methods for Nonlinear Boundary Value Problems," Communications of the ACM, 12(6), 327-330.

[7] SASTRY S.S, "Introductory Methods of Numerical Analysis."Prentice-Hall of India Private Limited, New Delhi.

[8] AHLBERG, J. H., NILSON, E. N. and Walsh, J. H.(1967): "The Theory of splines and their applications", Academic Press, N. Y.

[9] ALBASINY, E. L. AND HOSKINS, W. D.(1969), "cubic spline solutions to two-point boundary value problems", Comp. J. Vol. 12 p. 151.

[10] BERDYSHEV V.I.; SUBBOPIM JU .N.(1979): "Numerical Methods of Approximation of functions" (Russian). Sverdlovsk. Ural.Publ.House, 118pp 